\title{
An Investigation of Dextrous Manipulation Planning using C-Functions and Stability Functions
}

\author{
A.O Farahat
}

\author{
Aerospace Engineering Dept. \\ Texas A\&M University \\ College Station, Texas 77843
}

\begin{abstract}
The purpose of this paper is to investigate quasi-static dextrous manipulation using Cfunctions and stability functions and motion mode functions. In particular we consider the planning problem where we start with an object in an initial grasp configuration and attempt to find a joint trajectory to manipulate the object to a desired configuration. A central theme in this paper is the presumed lack of knowledge of the coefficient of friction.For a given joint trajectory we show how to establish upper bounds for the coefficient of friction below which the motion is qualitatively the same as in frictionless motion. We also show how to obtain upper bounds on the control error from a nominal joint trajectory deviation of control inputs from the nominal value below which the motion is qualitatively the same. Contact forces and joint torques for proper manipulation are also computed.
\end{abstract}

\section{Introduction}

The field of robot motion planning encompasses a wide range of problems, all of which can be stated as two-point boundary value problems; given the initial and goal states of the system, determine the control inputs that will effect the desired state change. When there is state uncertainty, then the solution must transform the sytem state from any point in the set of intial possible states into some point in the set of goal states. In this paper we restrict our attention to deterministic problems. The difficulty in solving these problems is correlated to the system's constraints. For example, repositioning and reorienting a manipulator's end effector in an uncluttered workspace is quite simple [1]. However, performing the same task amidst obstacles is quite difficult, since the manipulator's maneuver must satisfy geometric constraints to avoid collisions $[2,3,4,5,6,7]$. Problems involving contact are more difficult to solve than those without contact, owing to the fact that in addition to geometric constraints,

\author{
J.C Trinkle
}

a model of contact behavior must be satisfied. The simplest contact models assume that two objects are in contact; one object fixed in space and the other is firmly attached to a manipulator's end effector $[8,9]$. In this situation, one must consider the possibility of jamming (also called friction lock), but the stability of the grasped object is taken for granted. The dextrous manipulation planning problem provides additional complications. The object is "grasped" through contacts with a number of independent manipulators or "fingers." The problem to be solved can be stated as: given initial and goal grasps, determine the joint trajectories which, if executed, would accomplish the desired change of grasp. Since in dextrous manipulation planning problems, contacts between the object and the hand can break, stability conditions must explicitly be enforced at all times.

Our experience indicates that dextrous manipulation planning under the assumption of frictionless contacts is easier than when friction is considered $[10,11]$ - This is due to the fact that the contacts of a manipulated frictionless object are always sliding regardless of the direction of motion of the joints of the hand. However, when friction is considered, interplay among the geometry, joint velocities, and the coefficients of friction can cause some contacts to roll and others to slide. Thus in the frictional case there are more planning parameters. Since planning methods amount to searching the space of relevant parameters, the search time is typically exponential in the number of parameters. Therefore planning in a frictionless world would be desirable if one could subsequently execute the plan in a frictional world. In this paper, we consider a restricted frictionless dextrous manipulation planning problem. Our objective is to determine regions or cells in configuration space in which frictionless manipulation is stable and geometrically admissible. Then, determine the range of coefficient of friction, for which execution of the frictionless plan will result in the planned motion despite the presence of friction 


\section{Model of the quasi static Frictionless Mechanics}

\subsection{C-Space Formulation of the problem}

The frictionless manipulation planning problem was formulated by Trinkle in [12] in terms of C-space variables utilizing the concept the concept of contact formations [17]. A Contact formation is a qualitative description of the grasp that denotes the contacts between the fundamental elements of objects :vertices, edges, and surface patches. For the planar example shown in figure (1)vertex " 1 " is in contact with finger " 1 " vertex " 3 " is in contact with finger " 2 " and vertex " 5 " is in contact with the palm. We define contact formation " 1 ", denoted by CF1, as the contact formation of figure " 1 ". In C-Space, each each contact formation defines a CF-cell.Since the initial and goal cells are not necessarily in the same CF-cell, we have two planning problems ;an inter-cell planning problem and an intra-cell planning problem. The intra-cell planning can be stated as : given intial and final configurations of an object within the same CF-cell, determine the joint trajectories which if executed would accomplish the desired change of grasp. The inter-cell planning problem can be stated as :given any two configurations in two connected CF-cells $C F_{i}$ and $C F_{j}$ determine a sequence of joint trajectories that would cause the system configuration point to cross the boundary from $C F_{i}$ to $C F_{j}$. In this paper we only consider the intra-cell planning problem.

Here we present the geometric and stability constraints in terms of C-space variables for the CF of figure (1).

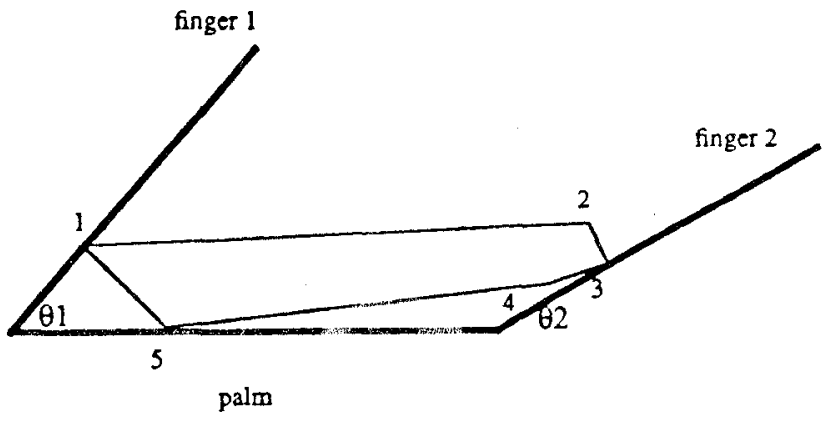

Figure 1: object

Consider the CF shown in figure 1, the geometric constraints in case of type (B) contact (vertex "a" of object is in contact with edge " $\mathrm{B}$ " of the obstacle ) are given by [13]

$$
\begin{aligned}
& \left\{V_{a}\left((q(1), q(2), q(3))-V_{a+1}(q(1), q(2), q(3))\right\} \cdot V_{n}^{B} \geq 0\right. \\
& \left\{V_{a}\left((q(1), q(2), q(3))-V_{a-1}(q(1), q(2), q(3))\right\} \cdot V_{n}^{B} \geq 0\right.
\end{aligned}
$$

where $V_{a-1}, V_{a}$ and $V_{a+1}$ are the position vectors of vertices "a-1", "a" and "a+1" of the object respectively. $V_{n}$ is the normal vector to edge " $B$ " of the obstacle and $q(1), q(2), q(3)$ are coordinates used to describe the position and orientation of the object with respect to a fixed coordinate frame.
For the two-fingered hand of figure (1) manipulating a polygonal convex object, inequalities (1),(2) imply that the object can be either in contact with the obstacle or breaking away from it. A negative value for the left- hand side would imply that the object is penetrating the hand which contradicts with the rigid body assumption. Inequality(1) must be satisfied at all contacts. For the three contact points configuration of figure(1) this gives ar set of three equality constraints and 6 inequality constraints. Let $F_{i, j}$ be the constraint $\mathrm{j}$ at finger $i$ where in general $F_{i, j}=\mathrm{F}\left(\mathrm{q}(1), \mathrm{q}(2), \mathrm{q}(3), \theta_{1}, \theta_{2}\right)$ where $i=1,2,3$ corresponds to finger 1, palm, finger 2 respectively $j=1$ corresponds to equality constraint and $\mathrm{j}=2,3$ corresponds to inequality constraint. This gives the following set of constraints

$$
\begin{aligned}
& F_{11}=0, F_{12} \geq 0, F_{13} \geq 0 \\
& F_{21}=0, F_{22} \geq 0, F_{23} \geq 0 \\
& F_{31}=0, F_{32} \geq 0, F_{33} \geq 0
\end{aligned}
$$

$\theta_{1}, \theta_{2}$, are the angular positions of fingers 1,2 respectively.

Due to the finite length of the fingers and the palm we also require that the contact point between finger " $B$ " and vertex " $a$ " remains physically on the edge and does not lie only on the supporting line of the finger or the palm.

$$
D_{b a} \leq D_{\max b}
$$

where $D_{b a}$ is the distance between contact point of vertex " $a$ " and a reference point " $b$ " on the finger measured along finger $\mathrm{B}, D_{\text {maxb }}$ is the maximum allowable distance from ref point " $b$ ". For the hand of figure " 1 " we shall assume that the fingers are very long and hence constraint (6) does not apply. However, we shall enforce constraint (6) for the contact with the palm.

The geometric constraints as given above are necessary but not sufficient conditions for a stable grasp. To have a stable,feasible grasp we must ensure that not only is the grasp geometrically feasible but that the forces acting on the object can balance any externally applied wrench.

Trinkle in [12] has shown that in order to have a stable grasp the normal contact forces dotted with their contact normals (pointing inwards with respect to the object ) must be greater than zero. This implies that while the object can be stability grasped using compressive contact load it can not be stability grasped using tensile contact force.

For the case of three contact points the normal contact forces are given by Trinkle in [12]

$$
\begin{array}{r}
c_{n}=\mathbf{W}_{n}^{-1} g_{e x t} \\
\mathbf{c}_{n} \geq 0
\end{array}
$$

where $W_{n}$ is the normal wrench matrix and $g_{\text {ext }}$ is an externally applied wrench. The wrench matrix should be nonsingular in order to keep the magnitude of the contact forces finite.

$$
\operatorname{Det}\left[\mathbf{W}_{n}\right] \neq 0
$$


The normal wrench matrix is related to $\mathrm{C}$-functions [12] as follows

$$
\mathbf{W}_{\mathbf{n}}=\left[\frac{\partial F_{11}}{\partial \mathbf{q}} \frac{\partial F_{21}}{\partial \mathbf{q}} \frac{\partial F_{31}}{\partial \mathbf{q}}\right]
$$

where $\frac{\partial F_{21}}{\partial \mathbf{q}}, \frac{\partial F_{21}}{\partial \mathbf{q}}, \frac{\partial F_{21}}{\partial \mathbf{q}}$ are $[3 \times 1]$ vectors.

\subsection{Identification of feasible grasps}

The set of geometric constraints (3), (4),(5),(6) and the force constraints (7),(8) must be simultaneously satisfied in order to have a geometrically valid, stable grasp. The parametrization of the position of the object using $q_{1}, q_{2}$ for the position of the center of gravity of object and $q_{3}$ for the orientation of the object

gives an object configuration space isomorphic to $\Re^{2} \times \mathcal{S}^{1} . \Re^{2}$ is the Euclidean space of dimension 2, $\mathcal{S}^{1}$ is the unit circle.

The configuration space of the two fingers is given by $\mathcal{S}^{2}$ (the unit sphere).

The overall configuration of the system is given by a five-dimensional manifold isomorphic to $\Re^{2} \times \mathcal{S}^{1} \times \mathcal{S}^{2}$. The configuration manifold $\mathcal{M}$ is a differentiable manifold and can be mapped onto compatible charts. Each compatible chart gives a unique parametrization of the manifold $\mathcal{M}$. While we have chosen to use the parametrization $q_{1}, q_{2}, q_{3}, \theta_{1}, \theta_{2}$ this parametrization is not unique. The constraint equations are in terms of trigonometric functions of the angular parameters $q_{3}, \theta_{1}, \theta_{2}$. and can be written as algebraic functions using a suitable change of coordinates. This is equivalent to using a compatible chart [14]. The real algebraic manifold obtained by making the change of variable

$$
t=2 \tan \frac{\theta}{2}
$$

where $\theta$ is any orientation angle $q_{3}, \theta_{1} \operatorname{or} \theta_{2}$ writing $w=\frac{t}{2}$ we get equation (11)

$$
\cos (\theta)=\frac{1-w^{2}}{1+w^{2}} \text { and } \sin (\theta)=\frac{2 w}{1+w^{2}}
$$

provides a parametrization of the algebraic manifold allowing application of the Collins [15] decomposition algorithm.

The geometric constraints $(3),(4),(5),(6)$ and the stability constraints $(7),(8)$ where applied to the hand of figure (1) in CF-1.Figure (2) shows the values of the fingers joint angles for which its possible to maintain geometrically valid and stable grasps. The contour lines in figure (2) correspond to different constraints being enforced. A contour line with value " 1 " indicates that the grasp is geometrically not feasible, a value of " 2 " indicates a singular wrench matrix, a value of " 3 " indicates that one or more elements of the wrench intensity vector are negative and a value of " 4 " indicates a stable, valid grasp

The intra-cell planning problem from an initial configuration " $\mathrm{I}$ " to a goal configuration " $\mathrm{G}$ " is reduced to finding a path connecting the two end points and is entirely contained in the region $\rho$ of valid configurations.

Figure(3) shows the valid configuration region $\rho$ drawn with a higher resolution. The region $\rho$ in figure 3 is not simply connected, indicating that within the region $\rho$ itself some configurations are not valid. In figure (3) two possible paths from an initial configuration to a final configuration are shown. Any valid path within the region $\rho$ should not cross any of the "holes" in that region.

\section{EFFECT OF FRICTION}

A mode of motion is a qualitative description of the motion of the object relative to the hand at the points of contact. At each contact point the object can slide,roll,or break away from the hand.The case of three sliding contacts is an example of a mode of motion. The type of relative motion (sliding,breaking,rolling)for the case of contacts with friction depends on the value of coefficient of friction at the contact point [16]. For the general case of $\mathrm{N}$ contacts, we might expect that all the coefficients of friction are different in which case we define the friction space referred to as the $\mu$-space to be the $\mathrm{N}$ dimensional Euclidean space of coefficients of friction at the $\mathrm{N}$ contacts. A particular mode of motion does not exist at only one point in the $\mu$-space but rather it defines a region in that space. Zeng describes the decomposition of the $\mu$-space into $\mathrm{r}$ such regions where the motion is qualitatively the same. She also derives the necessary conditions for the 3S motion ( three sliding contacts) as well as the RS (one rolling contact and one sliding contact).For the planner manipulation case there are only 3 independent velocity variables and only the $3 \mathrm{~S}$ or the RS modes of motion will satisfy the equations of motion, except in special cases. Region in $\mu$-space for RS motion The coefficient of friction cannot usually be determined with great accuracy. Therefore we consider the case where the coefficient of friction is essentially unknown. However, we only consider the case of $\mu_{1}=\mu_{2}=\mu_{3}\left(\mu_{1}, \mu_{2}, \mu_{3}\right.$ are the coefficients of friction at contact points $1,2,3$ respectively). While this assumption is not valid in general,for the case of same materials and loading conditions which do not vary considerably this assumption is justified.

For the case of contacts with friction equation (7) transforms to

$$
\begin{array}{r}
{\left[\mathbf{W}_{n}-\mathrm{W}_{t} \boldsymbol{\Xi}_{\mu}\right] \mathrm{c}_{n}=-\mathrm{g}_{\text {ext }}} \\
\text { Subject to } \mathrm{c}_{n} \geq 0
\end{array}
$$

where $\mu$ is the unknown coefficient of friction (the same for all three contacts)

$$
\begin{array}{r}
\mathrm{D}=\left[\mathrm{W}_{n}-\mathrm{W}_{t} \Xi \mu\right]^{-1} \\
\mathrm{c}_{n}=-\mathrm{Dg}_{\text {ext }} \\
\text { Subject to } \quad \mathrm{c}_{n} \geq 0
\end{array}
$$

where $\Xi$ is given by equation (22).

Substituting (14) into equation (15) gives the elements of the wrench intensity vector as a fraction of 
a quadratic polynomial $\alpha_{i}(\mu)$ and a cubic polynomial $\beta_{i}(\mu)$.

$$
\begin{array}{r}
c_{1 n}=\frac{\alpha_{1}\left(\mu^{2}\right)}{\beta\left(\mu^{3}\right)} \\
c_{2 n}=\frac{\alpha_{2}\left(\mu^{2}\right)}{\beta\left(\mu^{3}\right)} \\
c_{3 n}=\frac{\alpha_{3}\left(\mu^{2}\right)}{\beta\left(\mu^{3}\right)} \\
\text { Subject to } c_{1}, c_{2}, c_{3} \geq 0
\end{array}
$$

For all valid configurations the system of algebraic inequalities is satisfied for the frictionless case. In fact, for the case of frictionless contacts, equation (13) gives equation (8) which when augmented with geometric constraints (the geometric constraints are the same for the case of frictionless contacts and contacts with friction) defines the valid configurations. The Collins [15] decomposition or an approximate cell decomposition method should be used to solve equation $(15),(16),(17)$ for the general case where the coeffcients of friction are different. However, for the case of equal coefficients of friction since we know that $\mu$ $=0$ satisfies the system of equations (15),(16),(17) it is sufficient to determine the first change of sign for the system $(15),(16),(17)$. This is equivalent to finding the first positive real root for any of the equations of system (15),(16),(17).

To find the range of admissible values of $\mu$ it is necessary to determine the region $\nu$ in the $\mu$-space such that

$$
\begin{array}{r}
\{0,0,0\} \in \nu \\
\alpha_{1}, \alpha_{2}, \alpha_{3}, \beta \geq 0 \text { or } \\
\alpha_{1}, \alpha_{2}, \alpha_{3}, \beta \leq 0
\end{array}
$$

We define $\mu_{\max }$ to be the the smallest value of all such roots for a given configuration.

$$
\mu_{\max }=G L B\{\mu \mid \text { equation(12)is satisfied }\}
$$

If the coefficient of friction is greater than $\mu_{\max }$, then the motion of object will no longer be in the $3 \mathrm{~S}$ region , but will change to a different mode of motion (eg, RS motion).

Figure (4) gives the value of $\mu_{m a x}$ for the valid configurations of contact formation shown in figure (1). Figure (4) also shows two paths connecting and initial and goal configurations "I", "G" respectively. The two paths are entirely contained within the region of valid configurations, however path (1) allows a larger variation in the coefficient of friction.

A suitable measure of the coefficient of friction along the path eg(maximum

allowable $\mu_{\max }$,average $\mu_{\max }$ ) can be used as an input to an optimization procedure to select the optimum path from the set of all valid paths connecting "I", "G".

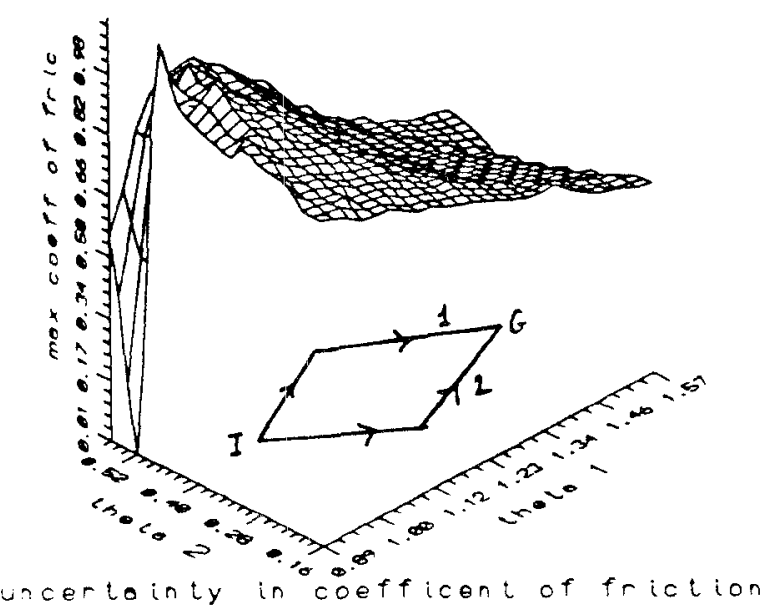

Figure 4:Max coefficient of friction

\section{Joint Velocity Sensitivity}

In section 3 we established the dependence of the $3 S$ type of motion on the coefficient of friction. The type of motion depends not only on the coefficient of friction, but also on the joint velocities and the grasp geometry. The motion of the non-breaking contacts can be divided into three types. The first type of motion corresponds to sliding along the tangent to the hand at point of contact. The second type of motion corresponds to sliding opposite the tangential direction at the point of contact.The third type of motion corresponds to a rolling contact. Figure (5) shows the coordinate frame at the point of contact.

In particular three possible types of motion are possible corresponding to

$\xi_{i}>0 \Rightarrow$ sliding motion in tangential direction

$\xi_{i}<0 \Rightarrow$ sliding motion opposite tangential direction

$\xi_{i}=0 \Rightarrow$ rolling motion. We define $\Xi$ to be the diagonal matrix with elements $\xi_{1}, \xi_{2}, \xi_{3}$. The relative velocity between the object and the hand at each of the three points of contacts is given by [16]

$$
[\xi]=\left[\mathbf{W}_{\mathrm{t}} \dot{\mathbf{q}}-\mathbf{J}_{\mathbf{t}} \dot{\theta}\right]
$$

Equation (20) can be rewitten as

$$
[\xi]=\left[\mathbf{W}_{\imath} \mathbf{W}_{n}^{-T} \mathbf{J}_{n} \dot{\theta}-\mathbf{J}_{i} \dot{\theta}\right]
$$


where $\mathbf{J}_{t}$ is the tangential Jacobian matrix, $\mathbf{J}_{n}$ is the normal Jacobian matrix and $W_{t}$ is the tangential wrench matrix For the case of the two-fingered hand with revolute joints the tangential component of the Jacobian matrix is identically zero.

$$
[\xi]=\left[\mathbf{W}_{t} \mathbf{W}_{n}^{-T} \mathbf{J}_{n}\right] \dot{\theta}
$$

For any choice of $\theta_{1}, \theta_{2}$ the motion of the object can be qualitatively described by $\left[\xi_{1}, \xi_{2}, \xi_{3}\right]$. The motion corresponding to our choice of $\dot{\theta}_{1}, \dot{\theta}_{2}$ is not restricted to a single value of angular velocities but is satisfied over a region in the $\dot{\theta}$ space. In particular we seek values for $\dot{\theta}_{1}, \dot{\theta}_{2}$ which give the same $\xi_{1}, \xi_{2}, \xi_{3}$ as a nominal value for angular velocity $\dot{\theta}_{\text {nom }}$ with components $\dot{\theta}_{\text {nom } 1}, \dot{\theta}_{\text {nom } 2}$ thus giving the same type of motion.

$$
\left[\begin{array}{ccc}
\xi_{1} & 0 & 0 \\
0 & \xi_{2} & 0 \\
0 & 0 & \xi_{3}
\end{array}\right]\left[\mathrm{W}_{t} \mathrm{~W}_{n}{ }^{-T} J_{n}\right]\left[\begin{array}{c}
\dot{\theta}_{1} \\
\dot{\theta}_{2}
\end{array}\right]>\left[\begin{array}{l}
0 \\
0 \\
0
\end{array}\right]
$$

We define $\theta_{\text {up }}, \theta_{\text {low }}$ to be the upper and lower limits of the region $\vartheta$ in the $\dot{\theta}$ space for which equation (22) is satisfied. We also define $\theta_{\max }$ to be

$$
\theta_{\text {max }}=\operatorname{Min}\left\{\left|\theta_{\text {nom }}-\theta_{\text {up }}\right|,\left|\theta_{\text {nom }}-\theta_{\text {low }}\right|\right\}
$$

$\theta_{\max }$ gives the maximum possible deviation in the angular velocity from a nominal trajectory for which the motion remains qualitatively the same provided that the coefficient of friction remains below $\mu_{\max }$ Figure (6) gives a plot of $\theta_{\max }$ at all feasible and stable configurations of contact formation 1. Figure (6) was calculated for a nominal trajectory $\theta_{1}=\theta_{2}$. Figure (6) also shows two possible paths connecting an initial and goal configurations "I", "G". The uncertainty in velocity can be used as an input to a an optimization procedure to select the optimum path from the set of all possible paths such that a suitable measure of uncertainty in velocity is optimized.

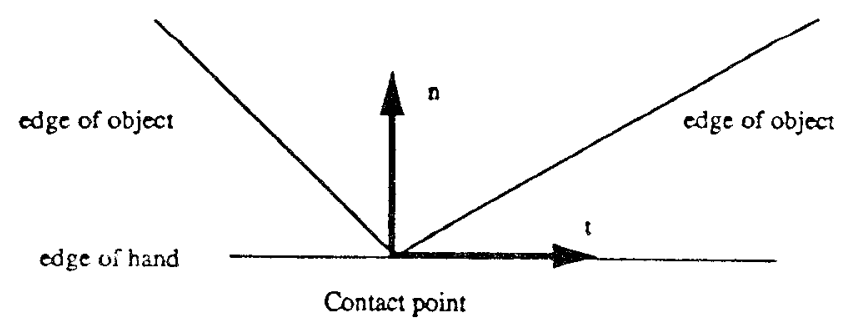

Figure 5:coordinate frame

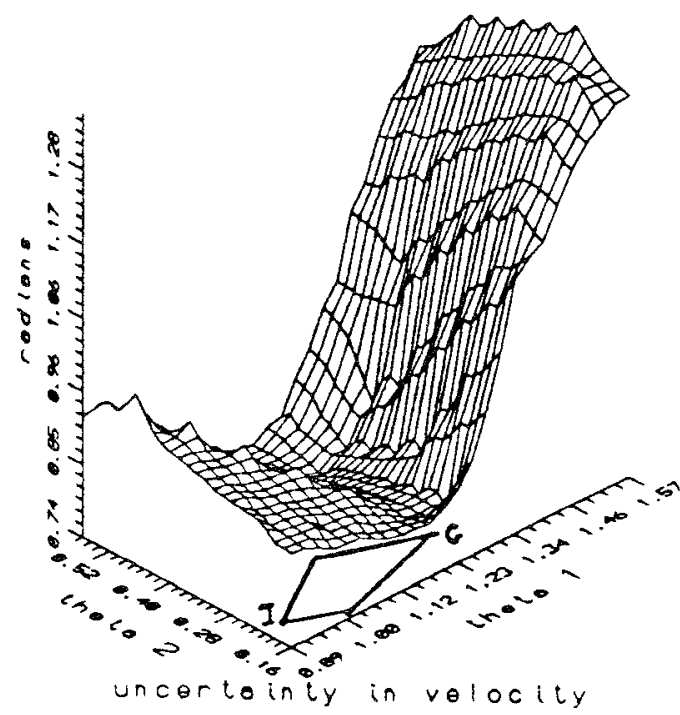

Figure 6:Velocity error bound

\section{Contact Forces}

The contact forces required to grasp the object are given by equation (8). Figure(7) shows the reaction forces at contact between palm and vertex 5 for all valid configurations of contact formation of figure(1).In a lot of situations it is desired to keep the contact forces as low as possible to avoid stresses in the object and to limit the control effort required to manipulate the object.Figure (7) gives two possible paths connecting initial and goal configurations "I", "G" .The value of the contact force can be used as an input to a an optimization procedure to select the optimum path from the set of all possible paths such that a suitable measure of the contact force is optimized. 


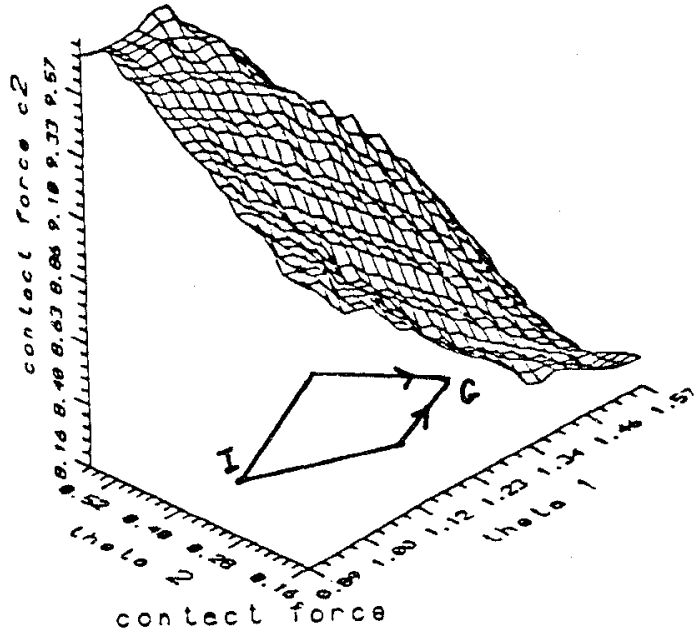

Figure 7 :Contact forces

\section{Joint Torques}

The Joint torques required to grasp the object are given by

$$
\tau=\mathbf{M}_{\text {hand }}(\theta) \ddot{\theta}+\mathbf{V}_{\text {hand }}(\theta, \dot{\theta})+\mathbf{G}(\theta)+\mathbf{J}_{\text {hand }}^{T} f
$$

Where $M$ is the generalized mass matrix, $G$ is the vector of gravitaional forces and $\mathrm{V}$ is the vector sum of centrifugal and Coriolis forces. For the quasi static approximation equation $(25)$ reduces to

$$
\tau=\mathbf{J}_{\text {hand }}^{T} f+\mathbf{G}(\theta)
$$

The values of the joint torques depend on the mode of motion and consequently on the angular velocities. Figure(8) show the torques required at joint 2 for all valid configurations of contact formation of figure(1) for a fixed angular velocities. Figure (8) gives two possible paths connecting initial and goal configurations "I", "G". The value of the joint torque can be used as an input to a an optimization procedure to select the optimum path from the set of all possible paths such that a suitable measure of the joint torques is optimized.

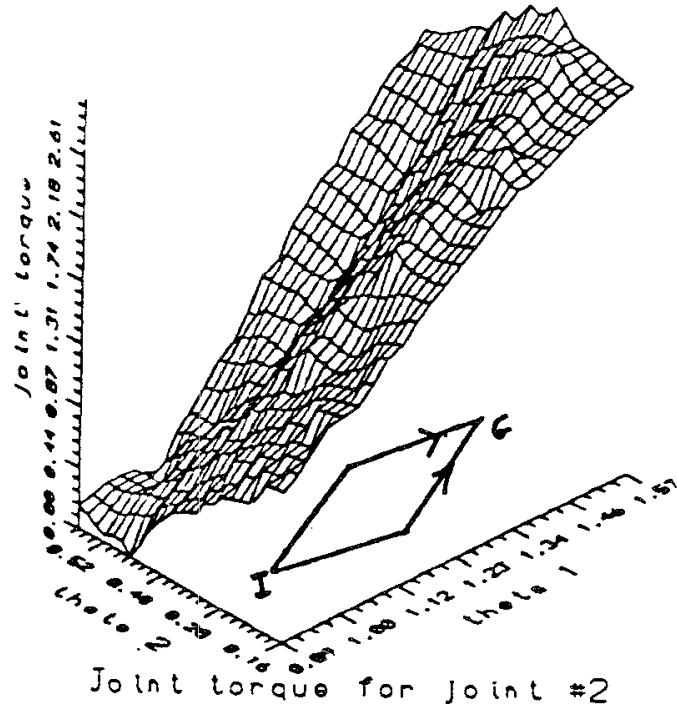

Figure $8:$ Joint torques

\section{Conclusion and future work}

The paper gives a framework for planning dextrous manipulation using $\mathrm{C}$-functions and stability constraints. We formulate the motion planning problem as a point navigation problem from an initial configuration to a final configuration in $\mathrm{C}$ - space. The identification of the region of feasible,stable grasps allows us to select a trajectory that satisfies certain constraints eg(coefficient of friction constraints, joint torques ....). The ability to give an upper bound on the variation of coefficient of friction has two major advantages. First, it allows us to plan the manipulation in the friction-less domain. The second major advantage of determining apriori the upper bound on the coefficient of friction is to be able to select the materials for both the hand and the object such that the coefficient of friction is always below a certain critical value. We also establish upper bounds on the variation of the angular velocities from a nominal value without changing the quality of motion. The joint torques and the reaction forces for a sample contact formation where calculated. Future plans include the parametrization of the configuration manifold using algebraic functions and applying Collins decomposition [15] to determine the region of feasible configurations. We also plan to use Collins decomposition [15] to decompose the $\mu$-space into regions having the same type of motion. The point navigation problem will be formulated as an optimal navigation problem and an optimal solution shall be given.

\section{Acknowledgement}

The first author would like to acknowledge the support of "USA/RC" The second author would like to 
acknowledge the support of the NSF. The findings and the results of this paper are the authors' and do not reflect the view point of the funding organizations.

\section{References}

[1] R. P. Paul, Robot Manipulators: Mathematics, Programming, and Control, MIT Press, Cambridge, MA, 1981.

[2] T.Lozano-Perez, "Handey:a robot system that recoginizes,plans and mainpulates," In Proceedings of 1987 IEEE Conference on Robotics and Automation, pp. 843-849, Raleigh, North Carolina, 1987.

[3] B. R.Donald, "A search algorithm for motion planning with six degrees of freedom," Artifical Intelligence, vol. 31, pp. 295-353, 1987.

[4] J. Canny, The Complexity of Robot Motion Planning, PhD thesis, Department of Electrical Engineering and Computer Science, MIT, 1987.

[5] C.K. Yap, "How to move a chair through a door," IEEE Transactions of Robotics and Automation, vol. RA-3, no. 3, pp. 173-181, 1987.

[6] J.Barraquanda, B.Langlois, and J.C.Latombe, "Robot motion planning with many of freedoms and dynamic constraints," In Robotics Research: The Fifth International Symposium, H. Miura and $\mathrm{S}$. Arimoto, editors, pp. 74-83, Cambridge, Massachusetts, 1990, MIT Press.

[7] P. Tournassound, "A strategy for obstacle avoidance and its application to multi-robot systems." In Proceedings of 1986 IEEE Conference on Robotics and Automation, pp. 1224-1229, San Francisco, CA, 1986.

[8] D.E.Whitney, "Quasi-static assembly of compliantly supported rigid parts," Journal of Dynamic Systems Measurments and Control, vol. 104, pp. $65-77,1982$.

[9] M.A.Erdman and M.T.Mason, "An exploration of senseorless manipulation," In Proceedings of International Conference on Robotics and $A u$ tomation, pp. 1569-1574. San Fransisco, CA, 1986 .

[10] C. Ang, On the Computability and Complexity of Contact Formations, Master's thesis, Department of Computer Science, Texas A\&M University, 1991.

[11] J. Trinkle and R. Paul, "An investigation of frictionless enveloping grasping in the plane," International Journal of Robotics Research, vol. 7, no. 3 , pp. 33-51, 1989.
[12] J. Trinkle, "On the stability and instantaneous velocity of grasped frictionless objects," to be. submitted to IEEE Transactions on Robotics and Automation,

[13] J.C.Latombe, Robot Motion Planning, Cluwer Academic Publishers, 1991.

[14] M.Spivak, Comprehensive Introduction to differential geometry, Publish or Perish Publication, 1972 .

[15] G. Collins, "Quantifier elimination for real closed fields by cylindrical algebraic decomposition," In proceeding of the Second GI Conference on Automata Theory and Formal Languages, volume 33, pp. 134-183. Springer Verlag, 1975.

[16] D. Zeng, On the Prediction of Quasi-Static Motion in the Presence of Uncertain Contact Friction, Master's thesis, Department of Computer Science, Texas A\&M University, 1991.

[17] R.S.Desai, On Fine Motion in Mechanical Assembly in Presence of Uncerainty, PhD thesis,Department of Mechanical Engineering, University of Michigan, 1988 . 

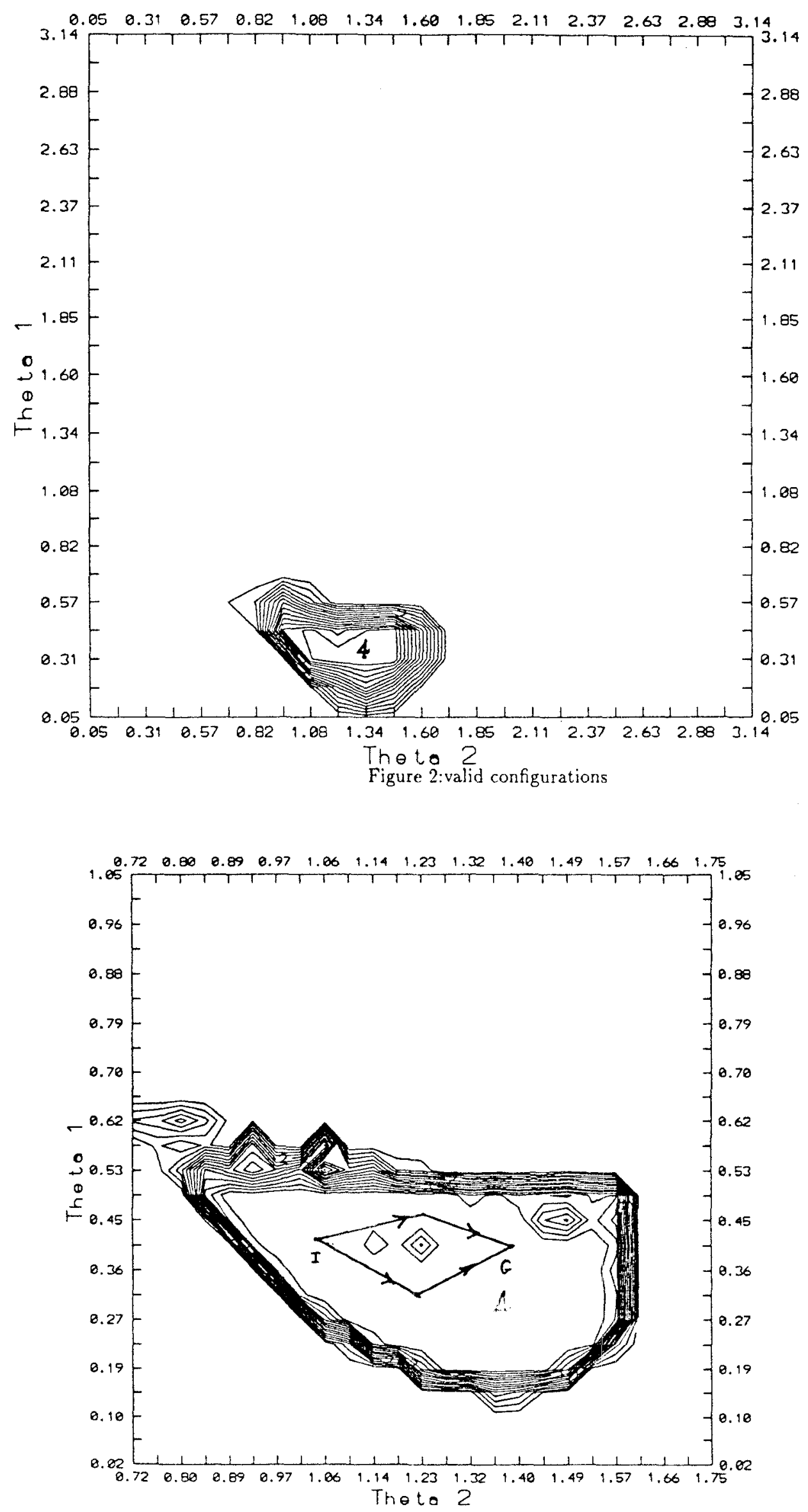

Figure 3:Intra Cell Planning 